\title{
Fracture Interaction during Temporarily Plugging Staged Fracturing
}

\author{
Bo Wang ${ }^{1}$, Fujian Zhou ${ }^{1}$, Jia Hu ${ }^{1}$, Liyang Gao ${ }^{2}$, Lishan Yuan ${ }^{1}$ and Yue Wang ${ }^{1}$ \\ ${ }^{1}$ Unconventional Natural Gas Research Institute, China University of Petroleum (Beijing), Beijing, CHINA \\ 2 Department of Petroleum Engineering, China University of Petroleum (Beijing), Beijing, CHINA \\ * Corresponding Author : zhoufj@cup.edu.cn \\ ORCID: 0000-0002-9050-3271
}

(First received 15 December 2017 and in final form 14 April 2018)

\section{Keywords \\ Fracturing \\ XFEM \\ Propagation \\ Simulation}

\begin{abstract}
Temporarily plugging staged fracturing technique has been successfully applied in unconventional reservoirs.Using XFEM-based CZM (cohesive zone model based on the extended finite element method), a 2D capturing stress interference and coupling fluid -solid model was established to investigate the process of the technique. The key points are as follows: (1) The diameter of the whole model was $1000 \mathrm{~m}$. The enriched zone is a square with the length of $200 \mathrm{~m}$. The whole model has 162299 nodes and 161901 elements. The quality of meshes meets the requirement of XFEM convergence. (2) plugging efficiency of diverting agents is simulated by changing injection rate. (3) the boundary condition of propped fractures is maintained by surface pressure, change of which simulates filtrate loss rate; (4) heterogeneity along horizontal well is simulated by setting various properties surrounding the subsequent fractures. The simulation results reveal that: (1) in-situ stress contrast affects the extent of the stress alteration zone, while in-situ stress magnitude impacts fracture length and width; (2) proper plugging efficiency will help to achieve even treated effect along the horizontal well; (3) with the augment of formation permeability, the extents of stress reversal and reorientation become small, thus subsequent fractures tend to propagate along the direction of maximum principle stress. (4) Young modulus and tensile strength have a great influence on propagation path, propagation rates, fracture length, and width.
\end{abstract}

\section{Introduction}

Multi-stage fracturing in the horizontal well is an indispensable method to develop unconventional reservoirs. The core of this technique is efficiently isolating the horizontal lateral to fracture the target formation one stage by one stage. While isolation tools have a great limitation when they are used in deep wells. In recent years, a new method has come into use, which is temporarily plugging staged fracturing by using self-degradable diverting agents. The key point is that self-degradable diverting agents replace the isolation tools [1-2].

During temporarily plugging staged fracturing, the previously fracture is plugged and propped by fracturing fluids and proppants, which exerts different degrees of stress-shadow effects. Fracture geometries during temporarily staged fracturing have not been simulated. In this paper, the effects of previous and propped fractures on the subsequent fractures were numerically simulated by using the extended finite element method based on the cohesive zone method (XFEM-based CZM). Key factors including the propped aperture of the previous fracture, in-situ stresses, formation permeability, Young's modulus and rock tensile strength were investigated.

\section{Mechanism of temporarily plugging staged fracturing}

Temporary plugging staged fracturing is mainly used in deep horizontal wells, for which the conventional mechanical isolation methods are dangerous and expensive. Fig. 1 gives the schematic of the process of temporary plugging staged fracturing (assuming $\mathrm{p}_{\mathrm{wf} 1}<\mathrm{p}_{\mathrm{wf} 2}<\mathrm{p}_{\mathrm{wf} 3}<\mathrm{p}_{\mathrm{wf} 4}<\mathrm{p}_{\mathrm{wf5}}$ ). During fracturing, injected fluids enhance the wellbore pressure gradually. When fluid pressure is larger than $p_{w f l}$, 
Frac. 1 begins to initiates and propagates. After Frac. 1 propagating to the desired distance, selfdegradable diverting agents are injected and form a tight impermeable slug, which plugs Frac. 1. And then the subsequent fracturing fluids continue to enhance wellbore pressure afresh. When wellbore pressure reaches $p_{\mathrm{wf} 2}$, Frac. 2 initiates and propagates. Repeat this process, and the whole lateral will be treated.

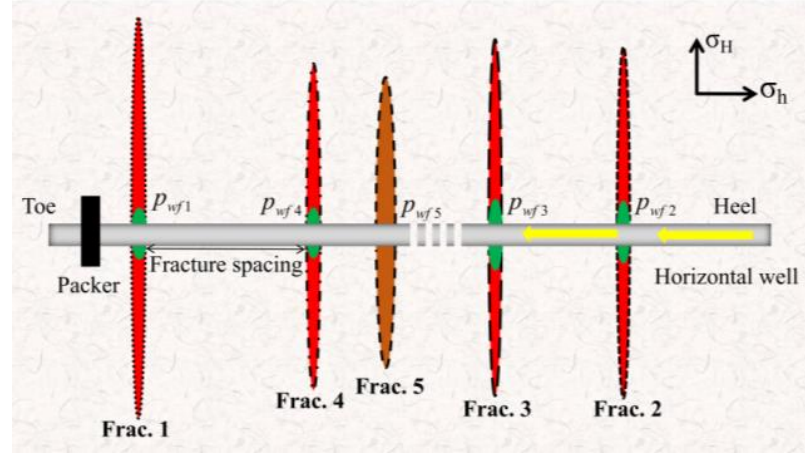

Figure 1. The process of temporary plugging staged fracturing (considering five fractures, actually, there are much more than five fractures). $p_{w f f}, p_{w f 2}, p_{w f 3}, p_{w f 4}$ and pwf5 are the initiation pressure of Frac. 1, Frac. 2,

Frac. 3, Frac. 4 and Frac. 5 respectively. (Assuming $\left.p_{w f 1}<p_{w f 2}<p_{w f 3}<p_{w f 4}<p_{w f 5}\right)$

\section{Fracture propagation model}

In this paper, a 2D plain strain model, coupling porous media deformation and fluid flow was established to perform the simulation studies. The extended finite element (XFEM) was used to describe fracture and maximum principal stress criterion was applied to determine the fracture propagation.

\subsection{XFEM Approximation}

XFEM was first proposed by Belytschko and Black[3]. In this approach, discontinuities (fractures) are allowed to cross the element and removing the requirement of re-meshing [4]. The existence of fractures is ensured by the special enriched functions. With the partition of unity enrichment, the displacement vector $\boldsymbol{u}$ is approximated by [5]

$$
\mathbf{u}=\sum_{I=1}^{N} N_{I}(x)\left[\mathbf{u}_{\mathrm{I}}+H(x) \mathbf{a}_{\mathrm{I}}+\sum_{\alpha=1}^{4} F_{\alpha}(x) \mathbf{b}_{\mathrm{I}}^{\alpha}\right]
$$

Where $N_{I}(x)$ are the usual nodal shape function; $u_{I}$ is the usual nodal displacement vector, $a_{I}$ is the nodal enriched degree of freedom vector, $H(x)$ is the discontinuous jump function across the fracture surfaces, $b_{I}^{\alpha}$ is the nodal enriched degree of freedom vector, $F_{\alpha}(x)$ is the elastic asymptotic crack-tip function.

\subsection{Cohesive zone method}

The cohesive zone method (CZM) consists of two parts: a damage initiation criterion and a damage evolution law. According to Mahdi Haddad [6], the maximum principal stress criterion used in XFEMbased CZM can be expressed by

$$
f=\left\{\frac{\left\langle\sigma_{\max }\right\rangle}{\sigma_{\max }^{o}}\right\}
$$

Where $f$ is the maximum principal stress ratio, $\sigma_{\max }^{o}$ is the maximum allowable principal stress [7].

The damage evolution law describes the rate at which the cohesive stiffness is degraded once the corresponding initiation criterion is reached. During fracture propagation, the Benzeggagh-Kenane (BK) criterion is used to determine the mixed-mode damage evolution [8]. The BK law model is described by

$$
G_{\text {equiv } C}=G_{I C}+\left(G_{I I C}-G_{I C}\right)\left(\frac{G_{I I C}+G_{I I I C}}{G_{I C}+G_{I I C}+G_{I I I C}}\right)(3
$$

Where $G_{\text {equivc }}$ is the computed equivalent fracture energy release rate; $\mathrm{G}_{\mathrm{IC}}$ is the Model I (tension failure) fracture energy release rate; $\mathrm{G}_{\mathrm{IIC}}$ is the Model II (shear failure under sliding) fracture energy release rate; $\mathrm{G}_{\text {IIIC }}$ is the Model III (shear failure under tearing) fracture energy release rate; in $B K$ roles, $\mathrm{G}_{\mathrm{IIC}}$ equals to $\mathrm{G}_{\mathrm{IIIC}}$.

\section{Model construction and verification}

\subsection{Model construction}

Using XFEM-based CZM, This paper presented a 2D plane strain model (Fig. 2), coupling rock deformation and fluid flow within fracture and porous media, to investigate the process of temporarily plugging staged fracturing. This model has 162299 nodes and 161901 elements (including pore pressure element (CPE4P) and truss element (T2D2)) with a very fine mesh around the fractures to improve the calculation accuracy. The diameter of the whole model is $1000 \mathrm{~m}$ and the enriched zone is a square with the length of $200 \mathrm{~m}$. Zerodisplacement and constant pore pressure boundary conditions during the simulation process are applied to the outer semicircle boundary "ACB". In order to reduce the computation cost, one half semicircle model is simulated on the symmetry of 
the diameter "AOB". Initial cracks are preset to simulate perforations and receive injected fluid. The investigation is carried out based on a threefracture array with fracture spacing of $20 \mathrm{~m}$ and the input parameters are listed in Table 1 (for a typical tight gas reservoir). We define the left fracture as Frac. 1, the middle fracture as Frac. 2, the right fracture as Frac. 3. During the simulation process, Frac. 2 propagates firstly and last for $1200 \mathrm{~s}$, after that, Frac. 2 is plugged by diverting agents and then Frac. 1 and 3 begin to initiate and propagate. The effects of Frac. 2 on the propagation geometries of Frac. 1 and 3 are investigated.

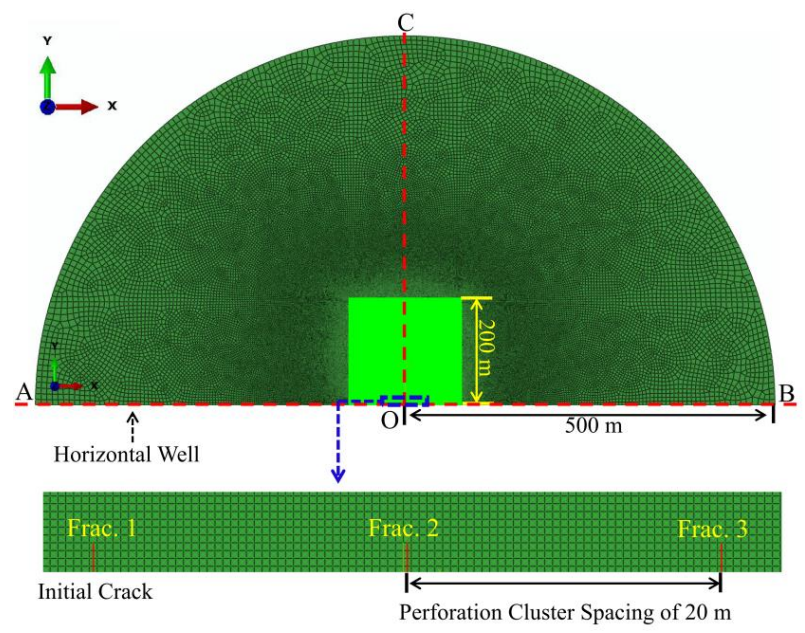

Figure 2. 2D Finite element model

Table 1. input parameters used in the simulation model

\begin{tabular}{|l|l|}
\hline PARAMETER & VALUES \\
\hline Young's modulus, $E(\mathrm{GPa})$ & 40 \\
\hline Poisson's ratio, $v$ (dimensionless) & 0.2 \\
\hline $\begin{array}{l}\text { Minimum principle horizontal stress, } \\
\sigma_{h}(\mathrm{MPa})\end{array}$ & 12 \\
\hline $\begin{array}{l}\text { Maximum principle horizontal stress, } \\
\sigma_{H}(\mathrm{MPa})\end{array}$ & 15 \\
\hline Initial pore pressure, $p_{o}(\mathrm{MPa})$ & 45 \\
\hline Injection rate, $Q\left(\mathrm{~m}^{3} / \mathrm{min}\right)$ & 4 \\
\hline Permeability, $k(\mathrm{mD})$ & 0.01 \\
\hline Void ratio, $\Phi($ dimensionless $)$ & 0.1 \\
\hline Fluid viscosity, $\mu(\mathrm{cp})$ & 10 \\
\hline $\begin{array}{l}\text { Damage initiation stress }(\text { equivalent } \\
\text { to rock tensile strength }), \sigma_{\text {omax }}(\mathrm{MPa})\end{array}$ & 2 \\
\hline $\begin{array}{l}\text { Critical energy release rate, } G_{I C} \text { and } \\
G_{I I C}(\mathrm{kN} / \mathrm{m})\end{array}$ & 30 \\
\hline Leakoff coefficient $\left(\mathrm{m}^{3} / \mathrm{kPa} \cdot \mathrm{s}\right)$ & $5.879 \times 10^{-10}$ \\
\hline
\end{tabular}

\subsection{Model Verification}

In this part, two fractures with the spacing of $10 \mathrm{~m}$ are simulated both with the model of this paper and the Unconventional Fracture Model (UFM) [9]. The input parameters are the same as presented by $\mathrm{Wu}$ [10] and the simulation results are given in Fig. 3. It shows that both of the fracture propagation path and the fracture width vs. length are in good agreement respectively.

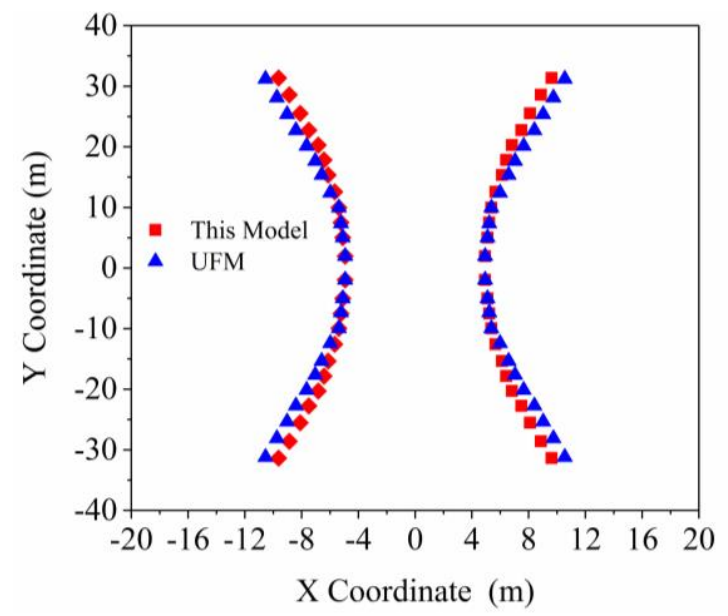

(a)

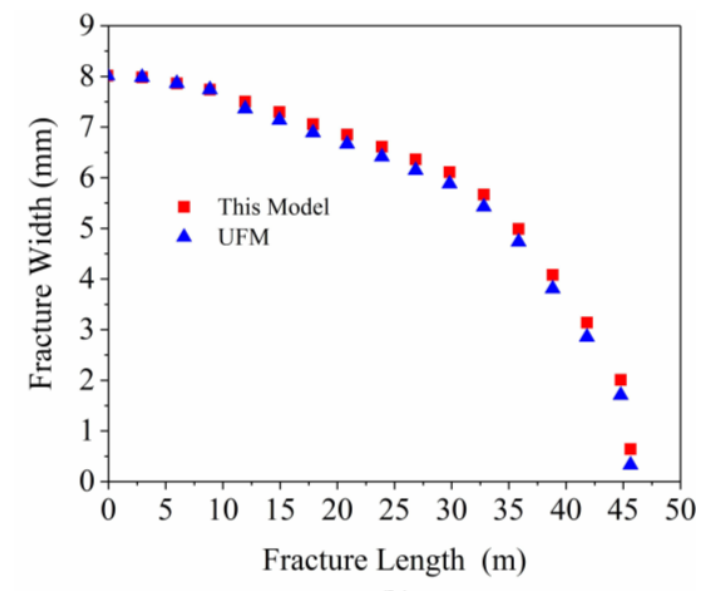

(b)

Figure 3. Comparison with published simulation results. (a) Trajectory comparison. (b) Fracture width comparison

\section{Results and discussion}

Based on the established model above, this section investigates the effects of important factors on the fracture geometries during temporarily plugging staged fracturing. These factors include plugging efficiency, propped aperture of the previous fracture, formation permeability, in-situ stresses, Young's modulus and rock tensile strength.

\subsection{Plugging efficiency}

High permeability lead to high fluid leak-off rate [11]. The effects of plugging efficiency were investigated by changing the injection rates of Fracs. 2. Four cases with the plugging efficiency of $50 \%, 60 \%, 90 \%$, and $100 \%$ were simulated 
respectively. The results (shown in Fig. 4) demonstrate that, given the total injection rate, Fracs. $1 \& 3$ propagate more maturely with the reduction of the injection rate of Frac. 2. Uniform fractures will enhance the simulation results. Therefore, during temporarily plugging staged fracturing, the optimal recipe of diverting agents should be used to ensure the perfect plugging of the previous fracture.

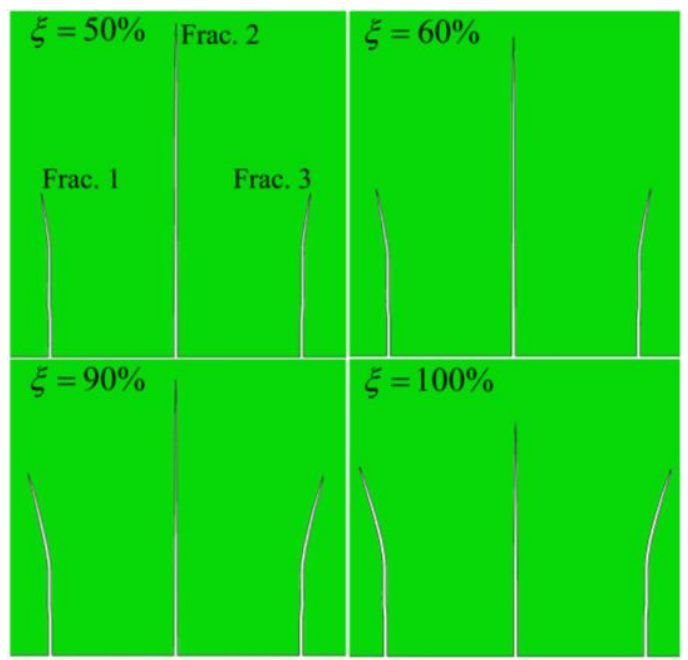

Figure 4. Propagation paths and apertures of the three fractures with various plugging efficiency of Frac. 2 (the previous fracture), 100-folds exaggeration of the apertures. $\xi$ is the plugging efficiency.

\subsection{Propped aperture of the previous fracture}

During temporarily staged fracturing, Frac. 2 (the previously created fracture) was plugged and propped, which generates rock deformation and alters the stress field. In this part, the opening of Frac. 2 was remained by exerting distribution pressure on the surfaces of Frac. 2. Four cases of $75 \%, 80 \%, 90 \%$ and $100 \%$ of the initial aperture propped were simulated. The simulation results (given in Fig. 5) illustrates that larger propped aperture of Frac. 2 generates bigger curvature and slightly smaller width of Fracs. 1\&3. Furthermore, Frac. 2 has lager length with the smaller aperture. This is because the larger aperture of Frac. 2 induces stronger stress shadow effects and increase the propagation resistance of Fracs. 1\&3. Thus the fluid pressure in Fracs. $1 \& 3$ is higher and the width is greater.

\subsection{Formation permeability}

Four cases with formation permeability of 0.0001 $\mathrm{mD}, 0.001 \mathrm{mD}, 0.01 \mathrm{mD}, 1 \mathrm{mD}$, respectively, were simulated to investigate the effects of previous and propped fracture on the propagation paths and width of subsequent fracture. The simulation results (Fig. 6) shows that the widths of the three fractures

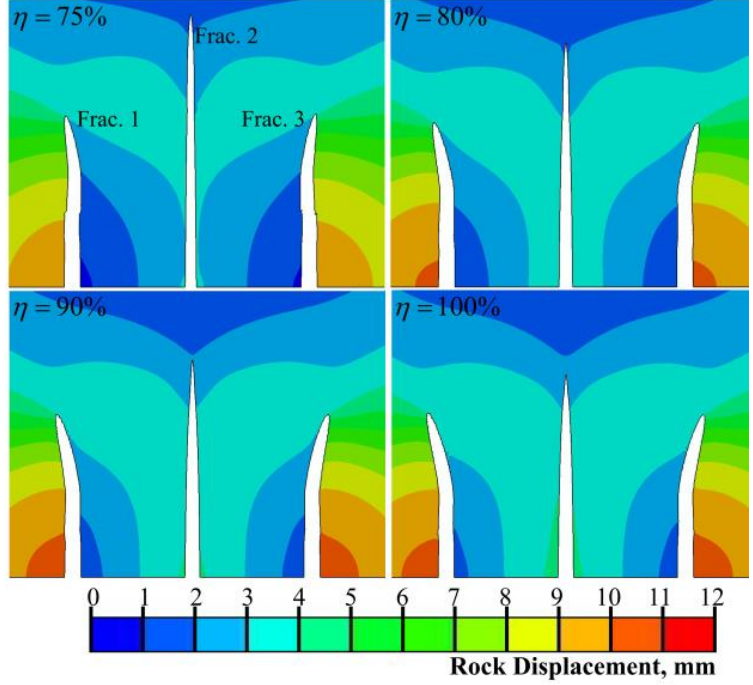

Figure 5. Propagation paths and apertures for the three fractures with varying apertures of Frac. 2,300-folds exaggeration of the apertures. nis the percent of the initial aperture of Frac. 2

decrease as formation permeability increase. While the lengths are on the contrary. This is because greater formation permeability generates higher leak-off rate, which reduces fracturing fluids remaining in fracture and decreases the fluid pressure. Lower fluid pressure lead to narrower fracture width and longer length. Therefore, in formation with high permeability, fracture interaction is not large during temporarily plugging staged fracturing.

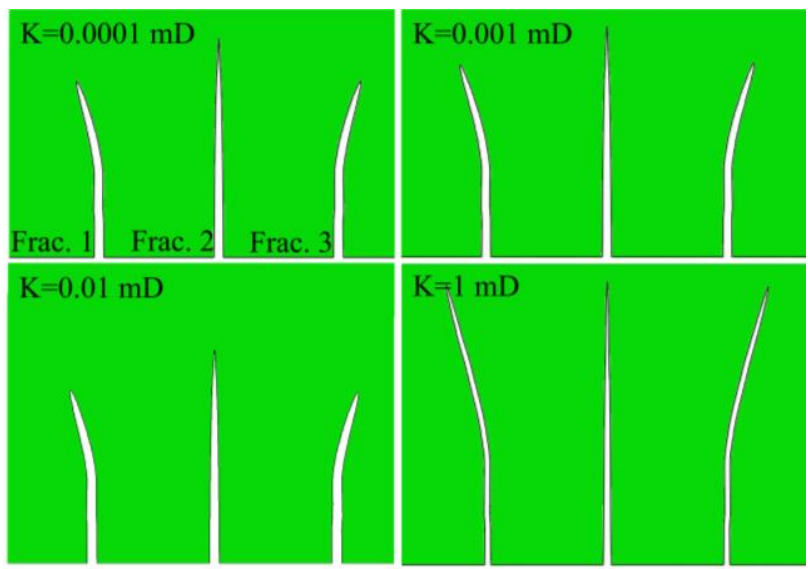

Figure 6. Propagation paths and apertures of the three fractures with varying formation permeability.300-folds exaggeration of the apertures. $K$ is the formation permeability.

\subsection{In-situ stress}

In this part, fracture propagation under the conditions of the different magnitude of in-situ stress and the horizontal differential stress are simulated. Fig. 7 gives the propagation geometries of the three fractures for the different magnitude of 
in-situ stresses and Fig. 8 presents that for various horizontal differential stress.

In Fig. 7, with the increase of the magnitude of insitu stresses, the widths of the three fracture become wider and the lengths of the three fracture become shorter. The reason is that high in-situ stress results in strong propagation resistance and increase the fluid pressure within the fracture. As stated above, high fluid pressure generates wider fracture width and shorter fracture length. In Fig. 8, with the increase of horizontal stress contrast, Fracs. $1 \& 3$ propagate more straightly with smaller fracture width. This is because, given the minimum horizontal stress, higher maximum horizontal stress more strongly restricts fracture propagation and then Fracs. $1 \& 3$ tend to propagate in the direction of the maximum horizontal principle stress with smaller curvature. Moreover, small fracture curvature brings about less propagation resistance and low fluid pressure, thus narrower width is obtained.

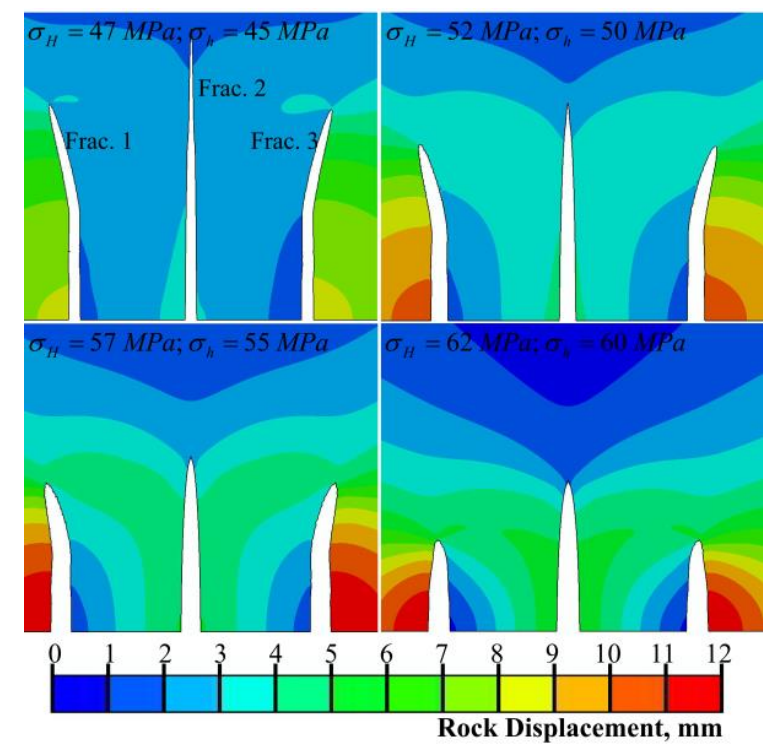

Figure 7. Propagation paths and apertures with different magnitude of horizontal stresses.300-folds exaggeration of the apertures. $\sigma_{H}$ is the maximum horizontal stress and $\sigma_{h}$ is the minimum horizontal stress.

\subsection{Formation heterogeneity}

In this part, the effects of formation heterogeneity were investigated simply by altering the mechanical properties of the rock surrounding Fracs. 1\&3 (the subsequent fractures). In Fig. 9, the larger the difference in rock tensile strength is, the more straightly Frac. $1 \& 3$ propagate. The reason is that larger rock tensile strength surrounding Fracs. 1\&3 brings about higher propagation resistance and

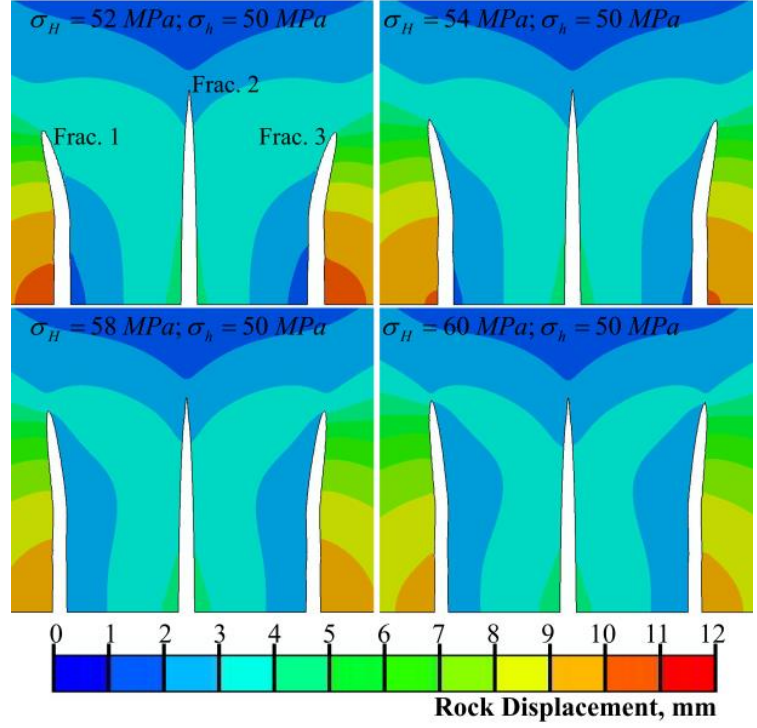

Figure 8. Propagation paths and apertures with different stress contrasts. $\sigma_{H}$ is the maximum horizontal stress and $\sigma_{h}$ is the minimum horizontal stress.

greater fluid pressure within Fracs. 1\&3. As stated above, greater fluid pressure generates more linearly fracture. In Fig. 10, with the increase of Young's modulus surrounding Frac. 1\&3, Frac. $1 \& 3$ propagate more deviously with narrower fracture width. This is because that rock with lower Young's modulus is soft and more likely to generate plastic deformation, which increases fracture propagation resistance. Thus, the fluid pressure within fractures is enhanced, which results in more straightly and wider fracture.

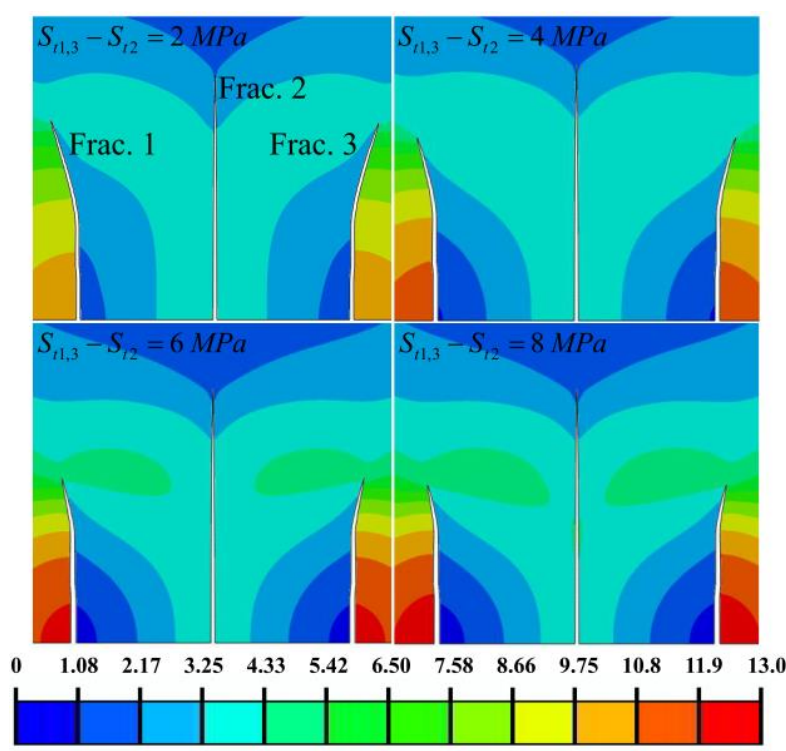

Rock Displacement, mm

Figure 9. Propagation paths for three fractures with different rock tensile strength .100-folds exaggeration of the apertures. $S t_{1,3}$ is the rock tensile strength surrounding Frac. $1 \& 3, S_{t 2}$ is the rock tensile strength surrounding Frac. 2. 


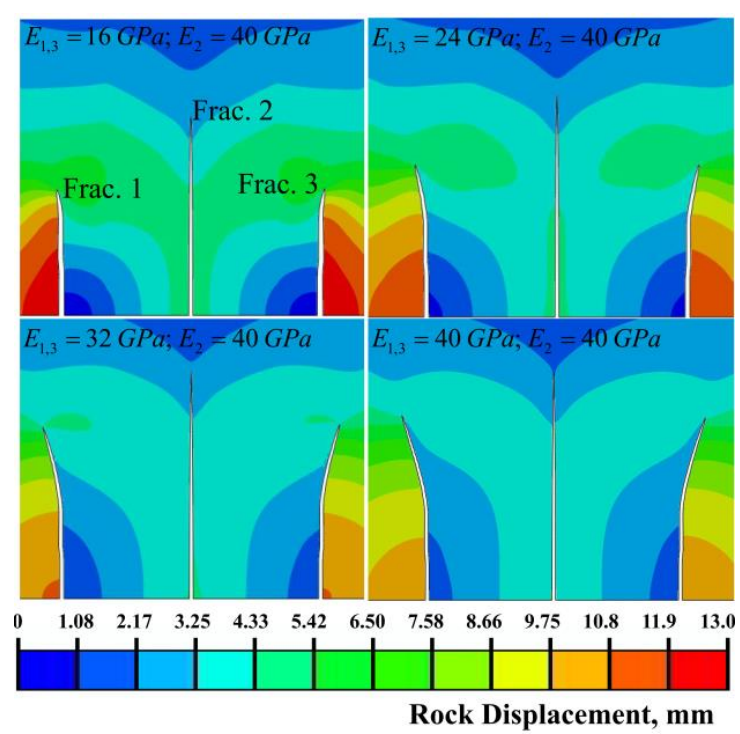

Figure 10. Propagation paths for three fractures with different Young's modulus surrounding Fracs. 1\&3, 100-folds exaggeration of the apertures. $E_{1,3}$ is the Young's modulus surrounding Frac. $1 \& 3, E_{2}$ is the Young's modulus surrounding Frac. 2.

\section{Conclusions}

Temporarily plugging staged fracturing is an effective method to develop unconventional reservoirs by using self-degradable diverting agents to replace isolation tools. To simulate the fracture geometries during temporarily staged fracturing, a 2D plain strain model using XFEM-based CZM was established. The effects of previously created and propped fracture on the propagation geometries of subsequent fracture under various conditions are investigated. These factors include the plugging efficiency, the propped aperture of the previous fracture, formation permeability, in-situ stresses, formation heterogeneity. Simulation results reveal that in-situ stress contrast affects the extent of the stress alteration zone, while in-situ stress magnitude impacts fracture length and width. Moreover, plugging the previous fracture efficiently will contribute to the uniform propagation of multiple fractures. Furthermore, formation permeability has little effects on the fracture propagation direction, while Young modulus and tensile strength have a great influence on propagation path, propagation rates, fracture length and width during temporarily plugging staged fracturing.

\section{Acknowledgement}

Authors thanks to Dassault Systemes Simulia Corparation for providing ABAQUS software program. This work is financially supported by National Key S\&T Special Projects (2016ZX05030-005) and National Key S\&T Special Projects (2016ZX05051).
This paper presented in " 4 rd International Conference on Computational and Experimental Science and Engineering (ICCESEN-2017)"

\section{References}

[1] Zhou, C., Wu, X., Li, H., Ren, Z., \& Xin, Y. (2013). Influence of in-situ stress distribution on selection of fracturing fluid backflow technology. Value Engineering, 130(1), 347-351.

DOI: 10.12693/APhysPolA.130.347

[2] Weddle, P., Griffin, L., \& Pearson, C. M. (2017, January 24). Mining the Bakken: Driving Cluster Efficiency Higher Using Particulate Diverters. Society of Petroleum Engineers. DOI:10.2118/184828-MS

[3] Belytschko, T., \& Black, T. (1999). Elastic crack growth in finite elements with minimal remeshing. International Journal for Numerical Methods in Engineering, 45(5), 601-620.

[4] Dahi Taleghani, A., \& Olson, J. E. (2014). How natural fractures could affect hydraulic-fracture geometry. SPE journal, 19(01), 161-171.

[5] Fries, T., \& Baydoun, M. (2012). Crack propagation with the extended finite element method and a hybrid explicit-implicit crack description. International Journal for Numerical Methods in Engineering, 89(12), 1527-1558.

[6] Haddad, M., \& Sepehrnoori, K. (2016). XFEMBased CZM for the Simulation of 3D MultipleCluster Hydraulic Fracturing in Quasi-Brittle Shale Formations. Rock Mechanics and Rock Engineering, 49(12), 4731-4748.

[7] ÖÖ. Karaçal. (2016). Computational material analysis of structural and hemodynamic model of coronary stent by $\mathrm{cfd} / \mathrm{fea}$ in computer aided mechanical engineering approach. Acta Physica Polonica, 130(1), 249-251.

DOI: 10.12693/APhysPolA.130.249

[8] Y. Özcanli, Çavuş, F. K., \& M. Beken. (2016). Comparison of mechanical properties and artificial neural networks modeling of $\mathrm{pp} / \mathrm{pet}$ blends. Acta Physica Polonica, 130(1), 444-446.

DOI: 10.12693/APhysPolA.130.444

[9] Weng, X., Kresse, O., Cohen, C.-E., Wu, R., \& Gu, H. (2011, November 1). Modeling of HydraulicFracture-Network Propagation in a Naturally Fractured Formation. Society of Petroleum Engineers. doi:10.2118/140253-PA

[10] Wu, R., Kresse, O., Weng, X., Cohen, C.-E., \& Gu, H. (2012, January 1). Modeling of Interaction of Hydraulic Fractures in Complex Fracture Networks. Society of Petroleum Engineers. doi:10.2118/152052-MS

[11] Shahri, M. P., Huang, J., Smith, C., \& Fragachán, F. E. (2017, August 28). Solid-Particulate Diverter Optimization: Coupling Perforation-Scale Particle Transport to Field-Scale Fracturing Simulation. American Rock Mechanics Association. 